\title{
Tratamiento mínimamente invasivo para fracturas toracolumbares
}

\author{
Matías Pereira Duarte, Gastón Camino Willhuber, Martín M. Estefan, Gonzalo R. Kido, Julio Bassani, \\ Matías G. Petracchi, Carlos A. Sola, Marcelo Gruenberg \\ Sector Patología del Raquis, Hospital Italiano de Buenos Aires, Ciudad Autónoma de Buenos Aires, Argentina
}

\begin{abstract}
RESUMEN
Introducción: Las técnicas mínimamente invasivas para tratar las fracturas toracolumbares se han desarrollado con el objetivo de reducir, al mínimo, la morbilidad perioperatoria. El objetivo primario de este estudio fue evaluar los resultados clínicos y radiológicos de una serie de pacientes con diagnóstico de fractura toracolumbar por causa traumática tratados con procedimientos mínimamente invasivos. Materiales y Métodos: Se llevó a cabo un análisis retrospectivo de los datos demográficos, radiológicos pre y posoperatorios, y funcionales posoperatorios mediante el índice de Oswestry y la escala analógica visual para dolor, en una serie de pacientes que cumplían los criterios de inclusión. Se registraron las complicaciones y la estancia hospitalaria. Resultados: Se analizaron 15 pacientes (10 hombres) con una edad promedio de 59 años y un seguimiento promedio de 32.9 meses. Los pacientes tenían un dolor promedio posoperatorio de 2/10 y un valor promedio del índice de Oswestry de 14/100. El análisis radiológico mostró un valor promedio preoperatorio de cifosis de $16^{\circ}$, que se corrigió a $10^{\circ}(p=0,01)$. La altura promedio de la vértebra comprometida fue de $18 \mathrm{~mm}$ en el preoperatorio y de $21 \mathrm{~mm}$ en el último seguimiento $(p=0,02)$. Hubo tres complicaciones $(20 \%)$ en tres pacientes. La estancia hospitalaria promedio fue de 9 días. Tres pacientes fueron internados nuevamente dentro de los 90 días de la cirugía. Conclusión: El tratamiento de las fracturas toracolumbares por causa traumática mediante técnicas mínimamente invasivas fue un procedimiento seguro y logró buenos resultados clínicos y radiológicos en el seguimiento a mediano plazo.
\end{abstract}

Palabras clave: Fractura toracolumbar; estabilización percutánea; cirugía mínimamente invasiva; fusión lumbar; artrodesis toracolumbar.

Nivel de Evidencia: IV

\section{Minimally Invasive Treatment for Traumatic Thoracolumbar Fractures}

\begin{abstract}
Introduction: Minimally invasive techniques for the treatment of thoracolumbar fractures have been developed to reduce perioperative morbidity. The primary purpose of this work is to evaluate the clinical and radiological outcomes in a group of patients with traumatic thoracolumbar fracture treated with minimally invasive procedures. Materials and Methods: Taking a group of patients within the inclusion criteria, we retrospectively analyzed demographic data, along with pre and postoperative radiographic measurements and postoperative functional scores, using the Oswestry Disability Index and the Visual Analog Scale for pain. We recorded the complications and the hospital stay. Results: 15 patients were analyzed (10 of them were male). The average age was 59 years, and the average follow-up was 32.9 months. The patients presented an average postoperative pain score of $2 / 10$ and an average Oswestry Disability Index score of $14 / 100$. The radiographic analysis showed an average preoperative segmental kyphosis value of $16^{\circ}$ that was corrected to $10^{\circ}(p=0.01)$. The preoperative average height for compromised vertebrae was $18 \mathrm{~mm}$, with an average height of $21 \mathrm{~mm}$ at the last follow-up $(p=0.02)$. There were 3 complications $(20 \%)$ in 3 patients. Hospital stay was 9 days long on average. Three patients were rehospitalized within 90 days after surgery. Conclusion: The treatment of traumatic thoracolumbar fractures by minimally invasive techniques was a safe procedure, with good clinical and radiological results in the medium term of follow-up.
\end{abstract}

Key words: Thoracolumbar fracture; percutaneous stabilization; minimally invasive surgery; XLIF; thoracolumbar arthrodesis.

Level of Evidence: IV 


\section{INTRODUCCIÓN}

Las fracturas de la región toracolumbar son las más frecuentes de la columna vertebral, ${ }^{1}$ a menudo, son causadas por lesiones de alta energía, incluidos los accidentes vehiculares y las caídas de altura. Estas lesiones generan dolor, deformidad y pérdida de la función. ${ }^{2}$ El objetivo del tratamiento consiste en la estabilización de las regiones traumatizadas y la descompresión si hay compromiso neurológico.

El abordaje posterior convencional es considerado el apropiado para pacientes con compromiso neurológico o sin él, y con fracturas por estallido y lesiones del complejo ligamentario posterior, con buenos resultados clínicos y radiológicos. ${ }^{3}$ Sin embargo, se ha asociado con complicaciones perioperatorias significativas, como pérdida sanguínea, infecciones y hospitalización prolongada. ${ }^{4}$ La desnervación y desvascularización de los músculos paraespinales durante este abordaje traen aparejada la consiguiente atrofia muscular. ${ }^{5}$ Esta desfuncionalización está asociada con la fisiopatología de dolor lumbar crónico., ${ }^{6,7}$

La fijación percutánea con tornillos pediculares y la cirugía mínimamente invasiva han evolucionado como una alternativa para el tratamiento de fracturas toracolumbares, con el objetivo de reducir, al mínimo, la lesión de los tejidos blandos ${ }^{8}$ y la morbilidad perioperatoria. ${ }^{9}$ La técnica percutánea, en particular, fue descrita inicialmente para el tratamiento de la enfermedad espinal degenerativa, ${ }^{10}$ requiere incisiones de $1 \mathrm{~cm}$ para cada tornillo y tubos dilatadores que disecan, de manera roma, la musculatura paraespinal. Al preservarla, limita la hemorragia, reduce la posibilidad de infección y disminuye el dolor posoperatorio. Asimismo, permite disminuir la estancia hospitalaria y acelerar la rehabilitación, y mejora los resultados funcionales posoperatorios. ${ }^{11-13}$ Disminuir la pérdida de sangre es particularmente importante en los casos de trauma espinal, así como en los pacientes geriátricos de alto riesgo. ${ }^{14}$

En nuestro Servicio, el tratamiento de las fracturas toracolumbares ha ido evolucionando hacia técnicas cada vez menos agresivas, y el abordaje convencional quedó limitado a los pacientes que así lo requieran. En los últimos años, hemos obtenido buenos resultados con artrodesis posteriores cortas de cuatro nivele ${ }^{15} \mathrm{o}$ ultracortas de dos niveles ${ }^{16} \mathrm{y}$, en la actualidad, llegamos a la implementación de técnicas mínimamente invasivas o percutáneas para su tratamiento.

El objetivo primario de este estudio fue evaluar los resultados clínicos y radiológicos de una serie de pacientes con diagnóstico de fractura toracolumbar de causa traumática tratados con cirugía mínimamente invasiva o percutánea en nuestro centro asistencial.

Como objetivo secundario nos propusimos estudiar la seguridad y la precisión en la colocación de los tornillos por vía percutánea.

\section{MATERIALES Y MÉTODOS}

Con la aprobación previa del protocolo de investigación por parte del Comité de Ética Profesional de nuestra institución, se llevó a cabo un análisis retrospectivo de los pacientes con diagnóstico de fractura traumática de la columna toracolumbar que cumplían con los siguientes criterios de inclusión: 1) >18 años de edad en el momento de la cirugía; 2) operado entre enero de 2012 y diciembre de 2017, en nuestra institución, 3) tratado mediante sistema de fijación percutánea de manera aislada (Figura 1) o asociada a artrodesis anterior mínimamente invasiva (Figura 2), 4) un seguimiento mínimo de 6 meses.

En este período, se trataron 129 fracturas vertebrales de causa traumática. Se excluyó a los pacientes: 1) con diagnóstico de fracturas cervicales, 2) tratados con un abordaje posterior convencional, 3) con vertebroplastia percutánea (Figura 3) y 4) sin historias clínicas electrónicas completas.

La información fue recolectada desde la historia clínica electrónica de nuestra institución. Se recabaron los datos demográficos de los pacientes. Se tomaron los datos de la evaluación clínica en el último seguimiento y el análisis de los parámetros radiológicos pre y posoperatorios, en el último control.

Se analizó clínicamente el dolor toracolumbar según la escala analógica visual (EAV) y mediante el Índice de Discapacidad de Oswestry (Oswestry Disability Index, ODI) ${ }^{17}$ El grado de compromiso neurológico, al ingresar y en la última consulta de seguimiento, según la escala de Frankel ${ }^{18}$ se obtuvo de la historia clínica.

Se evaluaron el mecanismo de lesión (alta o baja energía), el nivel de la vértebra fracturada, según la clasificación Magerl/AO ${ }^{19}$ y el ThoracoLumbar Injury Classification and Severity Score (TLICSS) ${ }^{20}$

Con respecto al procedimiento quirúrgico, se recabaron los siguientes datos: tiempos quirúrgicos de la cirugía percutánea y del procedimiento mínimamente invasivo (si lo hubo), cantidad de niveles estabilizados, cantidad de tornillos pediculares colocados por vía percutánea, días de espera entre la internación y el procedimiento, días de internación hasta el alta médica, nueva internación institucional no programada en los 90 días de la cirugía y su causa, complicaciones y el tratamiento indicado. 


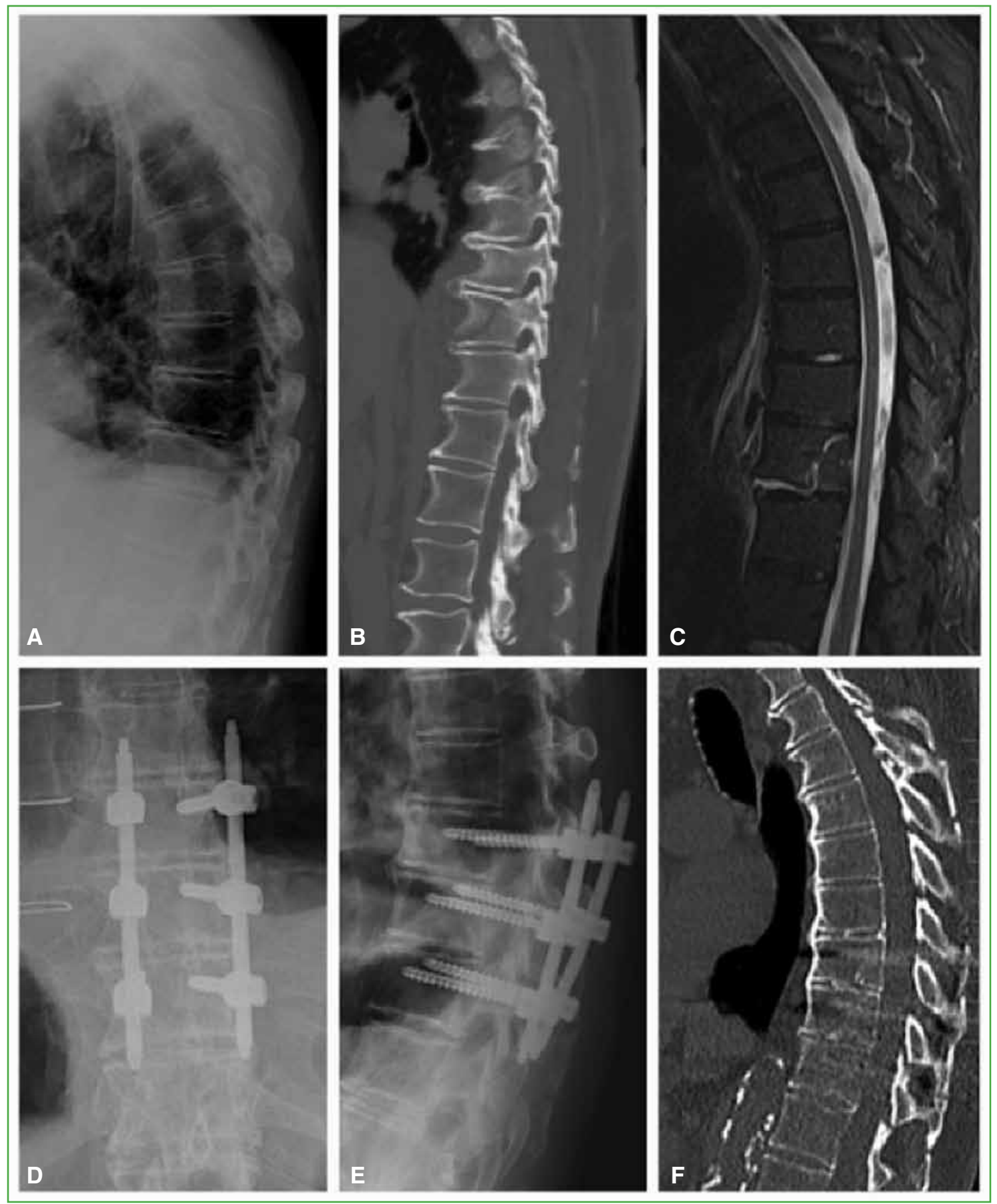

Figura 1. Paciente 7. Hombre de 81 años que sufre una caída de propia altura, con diagnóstico de fractura de $10^{\mathrm{ma}}$ vértebra torácica $\mathrm{AO} \mathrm{B} 3$. Al ingresar, se constata la lesión en la radiografía de perfil de la columna dorsal (A), en los cortes sagitales de la tomografía computarizada (B) y de la resonancia magnética en secuencia T2 (C). El paciente es operado al tercer día de ingresar mediante una estabilización percutánea desde T9 hasta T11 (D y E). F. Tomografía computarizada de control, a los 36 meses. Se observa la consolidación de la fractura sin pérdida de la altura ni cifotización del cuerpo vertebral. 


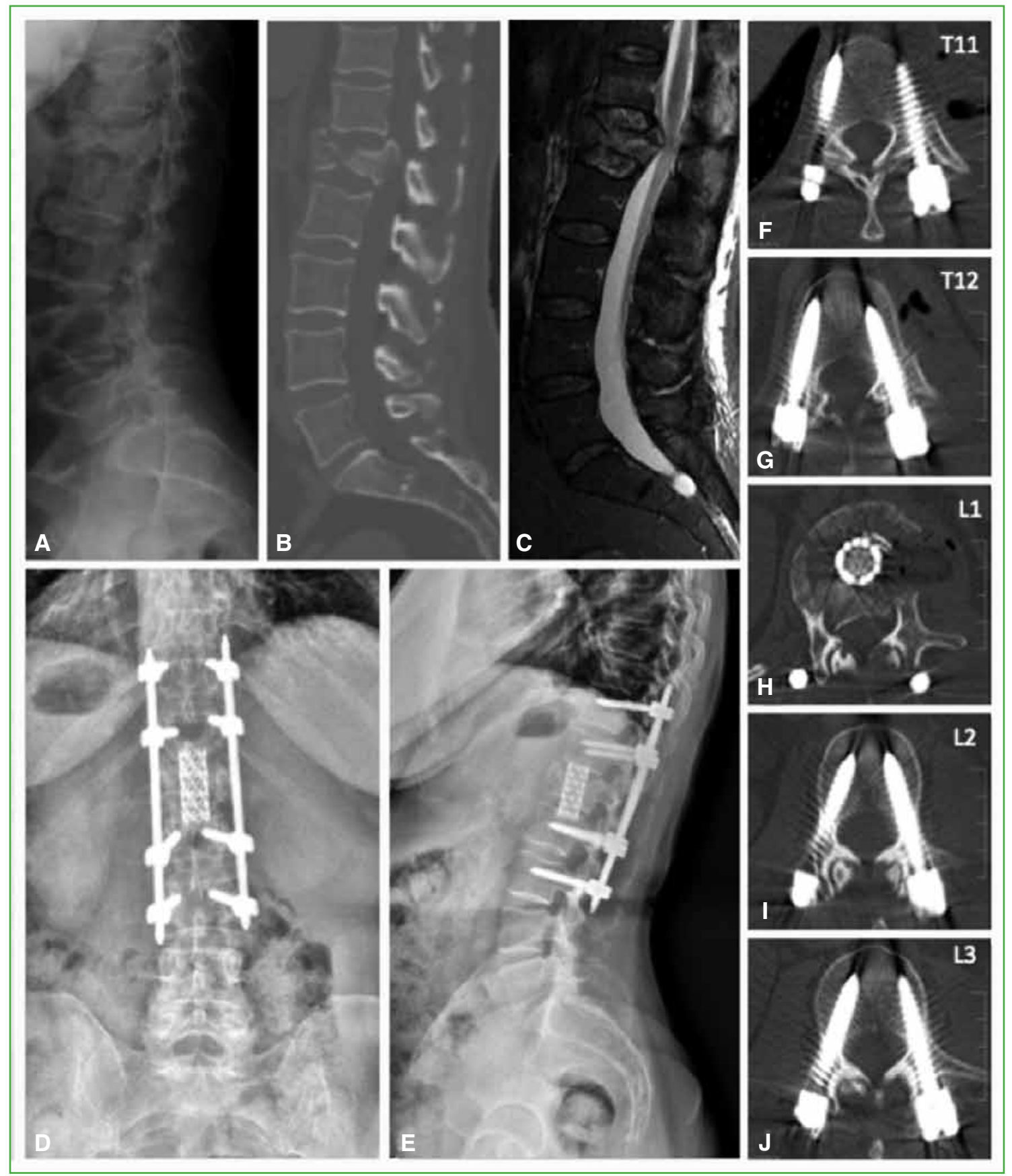

Figura 2. Paciente 6. Mujer de 60 años que sufre una colisión vehicular. A. Radiografía de columna lumbosacra de perfil. B. Tomografía computarizada, corte sagital. C. Resonancia magnética en secuencia T2. Se detecta una fractura AO A4 a nivel de la primera vértebra lumbar. Se observa el colapso del cuerpo vertebral con compromiso del muro posterior. Tiene una cifosis segmentaria de $15^{\circ}$ y una altura vertebral de $15 \mathrm{~mm}$. Al cuarto día de ingresar, es sometida a una estabilización percutánea de T11 a L3 (D y E). En un segundo tiempo quirúrgico y con la paciente clínicamente estable, se realiza una artrodesis mínimamente invasiva por abordaje lateral de T12 a L2, utilizando una celda Mesh e injerto óseo autólogo de cresta y costilla. F-J. Tomografía computarizada de control, a los 36 meses. Se observa la posición de los tornillos pediculares (5 en 1A y 3 en 1B, según Zdichavsky). 


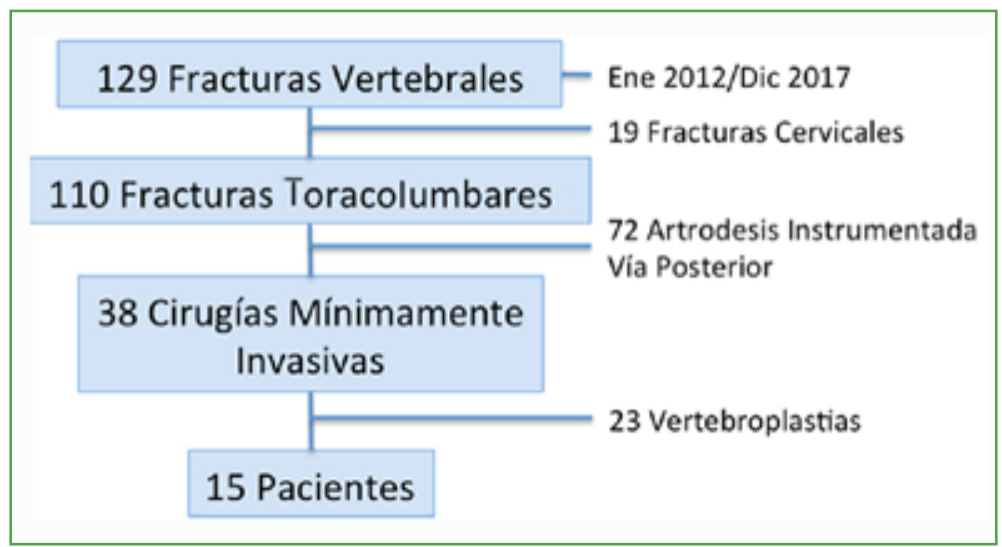

Figura 3. Diagrama de flujo en la selección de pacientes.

Constatamos los casos en los que se retiró el implante percutáneo y la causa, así como el tiempo de seguimiento hasta el retiro.

Para la estabilización de estas lesiones se utilizó el sistema de fijación percutánea multinivel CD Horizon Longitude $^{\mathrm{TM}}$ (Medtronic Sofamor Danek). Se insertaron tornillos pediculares poliaxiales con un diámetro de entre 4,5 y 7,5 mm, según el ancho del pedículo determinado mediante una tomografía computarizada (TC) antes de la operación. Los tornillos pediculares se insertaron de acuerdo con puntos de referencia anatómicos usando radioscopia intraoperatoria. Se evaluaron la seguridad y la precisión de la colocación de los tornillos pediculares mediante el análisis de los cortes axiales de TC a los 6 meses de la cirugía, con cortes de $3 \mathrm{~mm}$ utilizando la clasificación descrita por Zdichavsky y cols. ${ }^{21,22}$ (Figura 4).

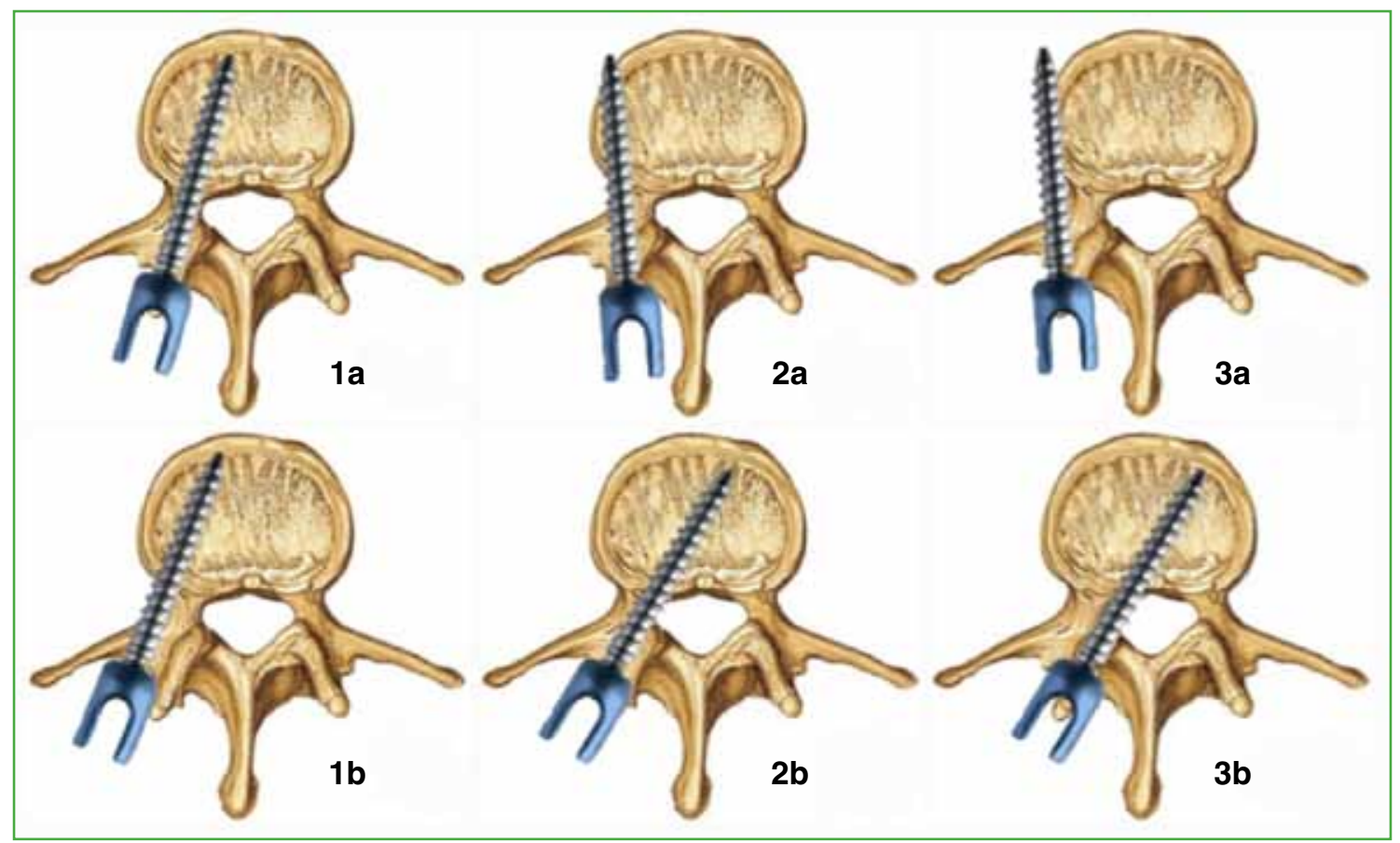

Figura 4. Clasificación de Zdichavsky y cols. para evaluar la posición de los tornillos. Las mejores opciones de posicionamiento son los tipos $1 \mathrm{~A}$ y $1 \mathrm{~B}$. En posición $2 \mathrm{~A}$ o $2 \mathrm{~B}$, debe evaluarse la estabilidad, pero generalmente no requieren revisión. En una mala posición $3 \mathrm{~A}$ y 3B, según la estabilidad o la posible irritación neurológica, se debe considerar la posibilidad de una revisión del tornillo. 
Los procedimientos mínimamente invasivos consistieron en la artrodesis intersomática por vía lateral ${ }^{23}$ de uno o dos niveles, con implantes o sin ellos. Se utilizó injerto local, de cresta ilíaca o de costilla para realizar la artrodesis anterior.

La indicación quirúrgica fue evaluada particularmente en cada paciente, todos fueron tratados mediante estabilización percutánea con tornillos pediculares. Además, se consideraron candidatos para una artrodesis anterior complementaria todos aquellos que cumplían algunos de los siguientes criterios: 1) fracturas A3 o A4 o B2 según la clasificación de Magerl/AO, con más de $20^{\circ}$ de cifosis segmentaria preoperatoria o compromiso ligamentario de la banda de tensión posterior o 2) fracturas con un puntaje $>4$ en la escala TLICSS.

Los pacientes fueron evaluados mediante radiografías, TC y resonancia magnética al ingresar en la Institución y con radiografías o TC en el último seguimiento. Se constataron los parámetros lumbopélvicos antes de la cirugía y después de ella, así como las mediciones referentes a la fractura: incidencia pélvica, inclinación pélvica, inclinación sacra, lordosis lumbar, cifosis torácica. Se determinaron la altura del cuerpo vertebral afectado y la cifosis segmentaria pre y posoperatoria, según la TC, se tomó como parámetro el extremo anterior del cuerpo vertebral en un corte sagital estricto y los platillos vertebrales superior e inferior de la vértebra involucrada, respectivamente ${ }^{24}$ (Figura 5).

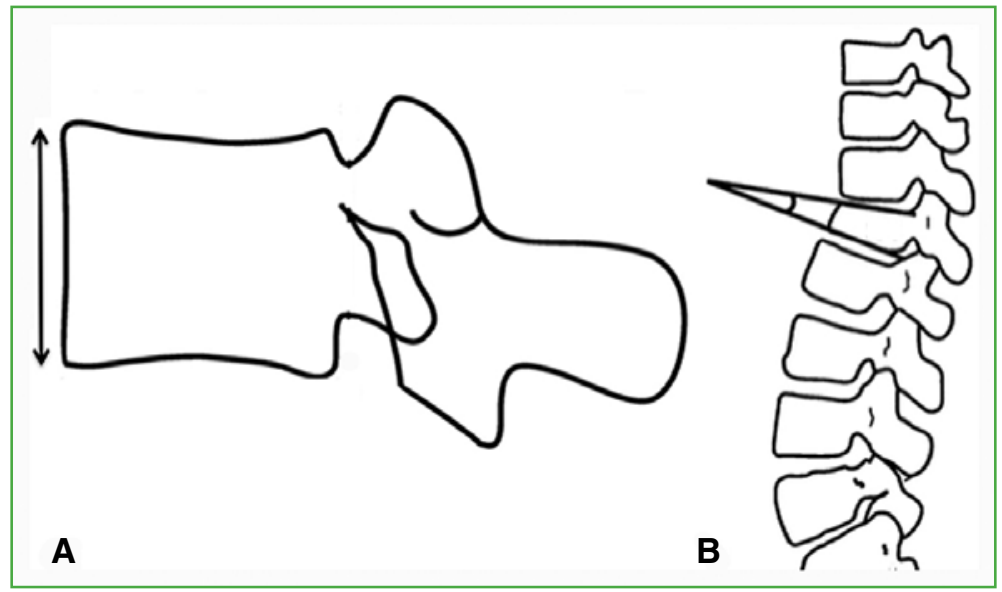

Figura 5. Esquema que representa los reparos anatómicos utilizados para realizar las mediciones tomográficas pre y posoperatorias de la altura corporal vertebral (A) y la cifosis vertebral segmentaria (B).

Se evaluó la consolidación de la fractura en la última consulta de seguimiento mediante TC, se la consideró satisfactoria si se observaba un puente óseo en la artrodesis intersomática, así como la reorganización de la trama ósea en la vértebra fracturada cuando se realizó el procedimiento percutáneo únicamente.

A fin de comparar las mediciones pre y posoperatorias de un mismo paciente se utilizó un test de T para muestras pareadas utilizando el programa Stata versión 13.

\section{RESULTADOS}

Entre enero de 2012 y diciembre de 2017, 15 pacientes con diagnóstico de fractura toracolumbar de causa traumática fueron sometidos a cirugía (Tabla 1): ocho por vía percutánea y siete combinando técnicas percutánea y mínimamente invasiva por medio de artrodesis intersomática por abordaje lateral. En cinco de estos siete pacientes, se utilizó un dispositivo intersomático con injerto óseo autólogo para otorgar un soporte anterior a la columna. En los dos restantes, solo se usó injerto óseo autólogo.

El promedio de edad fue de 59 años (rango 22-86). Diez eran de sexo masculino. El seguimiento promedio fue de 32.9 meses (rango 7-71) (Tabla 2).

Seis pacientes sufrieron traumatismos de baja energía (caída de propia altura); nueve, de alta energía (un aplastamiento por montacargas, cuatro caídas de altura y cuatro accidentes vehiculares) (Tabla 3). 
Tabla 1. Listado de pacientes intervenidos

\begin{tabular}{|c|c|c|c|c|c|c|c|c|c|c|c|c|c|c|}
\hline $\begin{array}{c}\text { Edad/ } \\
\text { Sexo }\end{array}$ & $\begin{array}{l}\text { Nivel de } \\
\text { fractura }\end{array}$ & $\begin{array}{l}\text { Clasifi- } \\
\text { cación } \\
\text { AO }\end{array}$ & TLICSS & $\begin{array}{l}\text { Cirugía } \\
\text { percutánea, } \\
\text { duración } \\
\text { (min) }\end{array}$ & $\begin{array}{l}\text { Cirugía } \\
\text { mininva- } \\
\text { siva, } \\
\text { duración } \\
\text { (min) }\end{array}$ & $\begin{array}{l}\text { Procedi- } \\
\text { mientos } \\
\text { asociados }\end{array}$ & $\begin{array}{l}\text { Niveles } \\
\text { fijados }\end{array}$ & $\begin{array}{l}\text { Instru- } \\
\text { menta- } \\
\text { ción }\end{array}$ & $\begin{array}{l}\text { Canti- } \\
\text { dad de } \\
\text { tornillos }\end{array}$ & $\begin{array}{l}\text { Compli- } \\
\text { caciones }\end{array}$ & $\begin{array}{l}\text { Trata- } \\
\text { miento }\end{array}$ & $\begin{array}{l}\text { Segui- } \\
\text { miento } \\
\text { (meses) }\end{array}$ & $\begin{array}{c}\text { EAV } \\
\text { poso- } \\
\text { perato- } \\
\text { ria }\end{array}$ & $\begin{array}{l}\text { ODI } \\
\text { pos- } \\
\text { ope- } \\
\text { rato- } \\
\text { rio }\end{array}$ \\
\hline $54 / \mathrm{M}$ & T12 & $\mathrm{A} 3 / \mathrm{B} 2$ & 6 & 150 & 240 & $\begin{array}{c}\text { Artrodesis } \\
\text { anterior } \\
\text { no instru- } \\
\text { mentada } \\
\text { T11-L1 }\end{array}$ & 4 & T10-L2 & 8 & $\begin{array}{l}\text { Intercos- } \\
\text { talgia }\end{array}$ & $\begin{array}{c}\text { Retiro del } \\
\text { material }\end{array}$ & 71 & 6 & 46 \\
\hline $64 / \mathrm{M}$ & L1 & A3 & 2 & 130 & & & 2 & T12-L2 & 6 & $\begin{array}{c}\text { Infección } \\
\text { de la } \\
\text { herida }\end{array}$ & $\begin{array}{l}\text { Desbri- } \\
\text { damiento } \\
\text { quirúr- } \\
\text { gico }\end{array}$ & 47 & 1 & 10 \\
\hline $58 / \mathrm{F}$ & L1 & $\mathrm{A} 4 / \mathrm{B} 2$ & 7 & 180 & 180 & $\begin{array}{c}\text { Artrodesis } \\
\text { anterior } \\
\text { T12-L2 }\end{array}$ & 4 & T11-L3 & 8 & $\begin{array}{c}\text { Infección } \\
\text { de la } \\
\text { herida }\end{array}$ & $\begin{array}{l}\text { Desbri- } \\
\text { damiento } \\
\text { quirúr- } \\
\text { gico }\end{array}$ & 44 & 2 & 12 \\
\hline $67 / \mathrm{F}$ & $\mathrm{T} 12$ & A3 & 2 & 90 & & & 4 & T10-L2 & 8 & & & 42 & 3 & 24 \\
\hline $61 / \mathrm{F}$ & L1 & A3 & 2 & 110 & & & 2 & T12-L2 & 4 & & & 41 & 0 & 12 \\
\hline $60 / \mathrm{F}$ & L1 & $\mathrm{A} 4$ & 2 & 110 & 140 & $\begin{array}{c}\text { Artrodesis } \\
\text { anterior } \\
\text { T12-L2 }\end{array}$ & 4 & T11-L3 & 8 & & & 36 & 3 & 12 \\
\hline $81 / \mathrm{M}$ & $\mathrm{T} 10$ & B3 & 7 & 140 & & & 2 & T9-T11 & 6 & & & 36 & 1 & 16 \\
\hline $72 / \mathrm{M}$ & L4 & $\mathrm{A} 4$ & 5 & 80 & 110 & $\begin{array}{l}\text { Artrodesis } \\
\text { anterior } \\
\text { L3-L5 }\end{array}$ & 2 & L3-L5 & 4 & & & 35 & 1 & 4 \\
\hline $34 / \mathrm{M}$ & L2 & A3 & 2 & 110 & 200 & $\begin{array}{l}\text { Artrodesis } \\
\text { anterior no } \\
\text { instrumen- } \\
\text { tada L1-L2 }\end{array}$ & 2 & L1-L3 & 4 & & & 33 & 3 & 14 \\
\hline $59 / \mathrm{M}$ & T12 & $\mathrm{A} 3$ & 2 & 105 & & & 2 & T11-L1 & 6 & & & 34 & 4 & 22 \\
\hline $86 / \mathrm{F}$ & $\mathrm{T} 11$ & B3 & 4 & 150 & & & 3 & T10-L1 & 8 & & & 23 & 4 & 16 \\
\hline $60 / \mathrm{M}$ & L1 & A3 & 2 & 140 & & & 4 & T11-L3 & 10 & & & 24 & 2 & 6 \\
\hline $57 / \mathrm{M}$ & L3 & A4 & 6 & 80 & 90 & $\begin{array}{c}\text { Artrodesis } \\
\text { anterior } \\
\text { L2-L4 }\end{array}$ & 2 & L2-L4 & 5 & & & 13 & 0 & 20 \\
\hline $49 / \mathrm{M}$ & L1 & A3 & 2 & 80 & & & 2 & T12-L2 & 6 & & & 8 & 3 & 2 \\
\hline $22 / \mathrm{M}$ & L2 & $\mathrm{A} 3 / \mathrm{B} 2$ & 7 & 165 & 105 & $\begin{array}{c}\text { Artrodesis } \\
\text { anterior } \\
\text { L1-L2 }\end{array}$ & 2 & L1-L3 & 5 & & & 7 & 1 & 4 \\
\hline
\end{tabular}

$\mathrm{M}=$ Masculino; F = Femenino; $\mathrm{T}=$ torácico $; \mathrm{L}=$ lumbar; TLICSS = ThoracoLumbar Injury Classification and Severity Score $;$ EAV = escala analógica visual, ODI = Oswestry Disability Index 
Tabla 2. Datos demográficos

$\mathrm{n}$

Edad (años)

59 (rango 22-86)

Sexo

Femenino

Masculino

10

5

Seguimiento (meses) 32.9 (rango 7-71)

Tabla 3. Resultados

\begin{tabular}{l|} 
Traumatismo \\
Baja energía \\
Alta energía
\end{tabular}

\begin{tabular}{|l|l|} 
Clasificación de Magerl/AO & 7 \\
A3 & 3 \\
A4 & 2 \\
B2 & 3 \\
B3
\end{tabular}

Clasificación TLICSS

$<4$

4

$>4$

Tiempo de la cirugía percutánea (min)

121 (rango 80-180)

Tiempo de los procedimientos mininvasivos (min)

160 (rango 90-290)

Estancia hospitalaria (días)

13 (rango 3-45)

TLICSS = ThoracoLumbar Injury Classification and Severity Score.

Doce fracturas (80\%) estaban a nivel de la transición toracolumbar: una en T11, tres en T12, seis en L1 y dos en L2. El resto se localizaban en T10, L3 y L4.

Según la Clasificación AO, siete fracturas fueron A3; tres, A4; dos, B3 y tres, B2 (2 con componente anterior A3 y uno con A4).

Los valores del TLICSS fueron: ocho fracturas $\leq 3$, una, 4 y seis, $\geq 5$.

El tiempo promedio de la cirugía percutánea fue de 121 min (DE $32 \mathrm{~min}$, rango 80-180). La duración promedio de los procedimientos mínimamente invasivos por vía lateral fue de $160 \mathrm{~min}$ (DE $57 \mathrm{~min}$, rango 90-240).

Se fijaron dos niveles en nueve pacientes, tres niveles en un paciente y, en cinco pacientes, cuatro niveles fueron estabilizados por vía percutánea.

En los 15 pacientes, se colocaron 96 tornillos de manera percutánea. Según Zdichavsky y cols., ${ }^{21,22} 75$ tornillos fueron colocados en posición 1A; 13, en posición 1B (mejores opciones de posicionamiento); dos, en 2A; cuatro, en 2B (en las que habitualmente se debe evaluar la estabilidad, porque existe violación de la pared pedicular) y dos, en 3B (existe la posibilidad de revisión del tornillo asociada a síntomas neurológicos o inestabilidad, ya que este se encuentra extrapedicular). No fue necesario una nueva intervención para reposicionar ningún tornillo.

El promedio de la estancia hospitalaria fue de 13 días (rango 3-45); el tiempo promedio de espera entre el ingreso y la cirugía fue de 3.7 días (rango 0-11) y el tiempo de rehabilitación desde la cirugía hasta el alta domiciliaria fue de 9.3 días (rango 3-41). Tres pacientes se internaron dentro de los 90 días de la cirugía: dos por infección de la herida quirúrgica de la vía percutánea, y requirieron desbridamiento quirúrgico sin retiro de la instrumentación. El otro paciente se volvió a internar por una descompensación de su insuficiencia cardíaca de base.

Se registraron tres complicaciones (20\%): dos infecciones de la herida (13\%) ya mencionadas, y un paciente que tuvo un dolor intercostal a los 16 meses de seguimiento y requirió el retiro de material (Tabla 1).

Todos tenían un puntaje de Frankel E al ingresar, excepto un paciente de 72 años que sufrió una fractura A4 de L4 con compromiso del $40 \%$ del canal raquídeo luego de una caída de propia altura, con un puntaje de Frankel D que evolucionó a $\mathrm{E}$ en el posoperatorio mediato. A este paciente se le realizó una estabilización percutánea de dos niveles con cuatro tornillos de L3 a L5 y una hemicorpectomía de L4 por vía lateral y artrodesis intersomática con celda rellena con injerto óseo autólogo por técnica mínimamente invasiva.

Todas las fracturas habían consolidado en el análisis radiológico al final del seguimiento, a los 32.9 meses (rango 7-71).

En dos de los 15 pacientes, se retiró el implante, en uno a los 16 meses por radiculalgia y, en otro, a los 5 meses por molestias cutáneas. 
Los resultados funcionales posoperatorios al final del seguimiento y los parámetros radiológicos evaluados se detallan en las Tablas 4 y 5 , respectivamente.

Tabla 4. Evaluación funcional

\begin{tabular}{|c|c|c|}
\hline & Media & Rango \\
\hline EAV posoperatoria & 2,27 & $0-6$ \\
\hline ODI posoperatorio & 14,57 & $2-46$ \\
\hline
\end{tabular}

$\mathrm{EAV}=$ escala analógica visual, $\mathrm{ODI}=$ Oswestry Disability Index .

Tabla 5. Parámetros radiológicos

\begin{tabular}{|lcccccc|} 
& Preoperatorio & DE & Posoperatorio & DE & p \\
\hline Incidencia pélvica $\left(^{\circ}\right)$ & 54,3 & 8,8 & - & & \\
Inclinación pélvica $\left(^{\circ}\right)$ & 21,2 & 10,7 & 18 & 6,9 & \\
\hline Inclinación sacra $\left(^{\circ}\right)$ & 37,1 & 11,1 & 35,4 & 6,5 & \\
\hline Lordosis lumbar $\left({ }^{\circ}\right)$ & 45 & 16,2 & 50,9 & 13,7 & \\
\hline Cifosis torácica $\left(^{\circ}\right)$ & 38 & 14 & 43 & 10,2 & \\
\hline Cifosis segmentaria $\left(^{\circ}\right)$ & 15,9 & 8,3 & 10,5 & 7,9 & 0,01 \\
\hline Altura vertebral $(\mathrm{mm})$ & 18 & 3,7 & 21,2 & 5 & 0,02 \\
\hline
\end{tabular}

\section{DISCUSIÓN}

Las tendencias actuales hacia técnicas quirúrgicas mínimamente invasivas representan un cambio importante en la práctica de la cirugía espinal. La instrumentación percutánea con tornillos pediculares en el traumatismo toracolumbar fue comunicada, por primera vez, en 2004, por Assaker, ${ }^{25}$ sin fallas de la construcción, mala posición de tornillos o aflojamiento de ellos. En estudios posteriores, se demostraron los beneficios de esta técnica comparada con el abordaje posterior convencional, entre ellos, un menor tiempo quirúrgico, estancia hospitalaria más corta, menos pérdida sanguínea, menor tasa de infección de la herida y mejores resultados funcionales y en la evaluación del dolor. ${ }^{9,21,23}$

Sin embargo, no se hallaron diferencias en cuanto al ángulo corporal y la altura vertebral anterior posoperatorios entre la cirugía posterior abierta convencional y la estabilización percutánea. ${ }^{9,26}$

Las indicaciones clásicas para el abordaje percutáneo incluyen fracturas con inestabilidad ósea transitoria que no requieren una reducción ni descompresión significativas. Se incluyen las fracturas tipo A de la clasificación de Magerl/AO, B1 con compromiso óseo de la banda de tensión posterior y aquellas con un TLICSS $<5 .{ }^{13,25}$

En nuestra serie, tratamos siete fracturas A3 de la clasificación de Magerl/AO, tres A4, dos B3 y tres B2 (2 con componente anterior A3 y uno con A4), que corresponden a ocho fracturas con un TLCISS $\leq 3$, una con 4 y seis, $\geq 5$. A pesar de que Vaccaro y cols. ${ }^{20}$ promueven el tratamiento conservador de las fracturas toracolumbares con un TLICSS $<4$, esta indicación es sumamente controversial. Los resultados clínicos a largo plazo en fracturas tipo estallido (A3 y A4) no parecen tener diferencias clínicamente significativas con ambos tipos de tratamiento, aunque los resultados radiológicos sean mejores en aquellos operados. ${ }^{27}$ Asimismo, el tratamiento quirúrgico de estas lesiones se relaciona con una mejoría del dolor a corto plazo y tiempos de retorno a las actividades laborales y de la vida cotidiana más rápidos ${ }^{28}$ En este estudio, un subgrupo de ocho pacientes con un TLICSS $<4$ fue operado, con su consentimiento y el de su familia quienes optaron por este método para evitar períodos prolongados de inmovilización, reposo y dolor junto con las potenciales consecuencias de estos. 
La combinación de fijación percutánea con técnicas anteriores de reconstrucción espinal mínimamente invasivas permite preservar las ventajas potenciales de este procedimiento. Esta combinación se indica cuando la fijación posterior aislada no es mecánicamente suficiente (Load Sharing Classification de McCormack >6), ${ }^{29}$ aquellas fracturas A3, A4 o B2 que tienen una compresión vertebral significativa que conduce a la pérdida de la altura del cuerpo vertebral y un vacío óseo anterior o aquellas con un TLICSS $>4$. Siete de nuestros 15 pacientes fueron estabilizados por una vía anterior adicional. Cuatro fracturas fueron clasificadas como A4 y tres como A3, tres de las cuales tenían un componente B2. El TLICSS promedio fue de 4 (rango 2-7).

Una de las ventajas comunicadas de las técnicas mínimamente invasivas es la tasa más baja de infección del sitio quirúrgico. Ni y cols. ${ }^{30}$ informaron una infección superficial en 36 pacientes con fijación percutánea, que fue tratada solo con antibióticos; Schmidt y cols., ${ }^{14}$ y Merom y cols. ${ }^{31}$ no detectaron infecciones en 76 y 10 pacientes, respectivamente; y Palmisani y cols..$^{32}$ comunicaron una sola infección que requirió el retiro de la instrumentación en 64 pacientes. Estos estudios ${ }^{14,30-32}$ informan una tasa baja de infección comparada con las reportadas para los procedimientos abiertos convencionales, que varían del 3,1\% al 10\%. ${ }^{33}$ En esta serie, hubo dos infecciones de heridas en 15 pacientes (13\%) (Tabla 2). Ambas requirieron desbridamiento quirúrgico y antibioticoterapia por vía intravenosa. Los gérmenes aislados fueron Staphylococcus epidermidis en un hombre de 64 años y Enterococcus faecalis en una mujer de 58 años. Los dos casos se resolvieron sin que fuera necesario retirar la instrumentación. Esta complicación ocurrió en los primeros casos, y consideramos que la escasa experiencia en el manejo del abordaje quirúrgico favoreció esta situación. Las incisiones más pequeñas no necesariamente son mejores, los bordes cutáneos sufren el traumatismo repetitivo durante el procedimiento y se desvitalizan con la distracción con el instrumental, lo que favorece la colonización y la infección. Cabe destacar que si la cantidad de pacientes es baja, puede influir en los resultados, sobrestimando la tasa de infección. No ocurrió esta complicación en el resto de la serie. Estos abordajes mínimamente invasivos también favorecen una estancia hospitalaria más corta ${ }^{90,30,34}$ debido a una rehabilitación más rápida. Merom y cols. ${ }^{31}$ hacen referencia a que los pacientes tratados por vía percutánea lograron deambular en uno o dos días, mientras que los tratados mediante un abordaje posterior convencional lo hicieron a los tres o cuatro días. En una revisión sistemática de 2015, Phan y cols. ${ }^{9}$ comunican que la estancia hospitalaria promedio para los procedimientos mínimamente invasivos en 279 pacientes con fracturas toracolumbares fue de 10.7 días. Nuestros pacientes permanecieron internados un promedio de 9 días después de la cirugía (rango 3-41), este último corresponde a una mujer de 60 años que tuvo una internación prolongada como consecuencia de un accidente de alta energía con lesiones en múltiples órganos. Este valor es comparable con los datos de otras series. ${ }^{9,34}$ Asimismo, tres de 15 pacientes (20\%) tuvieron reinternaciones no programadas dentro de los 90 días posoperatorios, dos por infecciones de la herida y uno por una descompensación de la insuficiencia cardíaca, cumplieron 40, 32 y 21 días de internación totales, respectivamente, en los tres primeros meses posoperatorios. No hemos hallado este dato reflejado en otros estudios similares.

En cuanto al análisis radiológico, se ha publicado que no existen diferencias estadísticamente significativas en relación con la cifosis pre y posoperatoria, y la altura del cuerpo vertebral pre y posoperatoria entre los pacientes tratados con un procedimiento mínimamente invasivo y los operados por el abordaje convencional. ${ }^{9,26}$ Pelegri y cols., ${ }^{35}$ y Ni y cols. ${ }^{30}$ reportan una cifosis promedio preoperatoria de $16^{\circ}$ y $18^{\circ}$, respectivamente, en sus series de pacientes tratados por vía percutánea; estas mejoraron $8^{\circ}$ y $9^{\circ}$ en promedio, al final del seguimiento, respectivamente. Por el contrario, Palmisani y cols..$^{32}$ informan una pérdida de corrección promedio de $4^{\circ}$ en 57 fracturas tipo A tratadas por vía percutánea con segmento corto. Refieren que esta pérdida fue mayor con los tornillos poliaxiales que con los monoaxiales.

En nuestra serie, los pacientes tuvieron una cifosis segmentaria promedio de $16^{\circ}\left(\mathrm{DE} 8^{\circ}\right)$, se corrigió y se mantuvo, en promedio, en $10^{\circ}\left(\mathrm{DE} 8^{\circ}\right)$ al final del seguimiento, esto representa una reducción y un mantenimiento promedio de $5^{\circ}$, lo cual fue estadísticamente significativo $(\mathrm{p}=0,01)$. De la misma manera, la altura de la vértebra comprometida fue, en promedio, de $18 \mathrm{~mm}$ (DE 3,7) cuando se decidió la cirugía, aumentó y se mantuvo en un promedio de $21 \mathrm{~mm}$ (DE 5) en el último seguimiento, lo que también resultó significativo $(\mathrm{p}=0,02)$.

$\mathrm{Al}$ analizar de manera diferencial aquellos pacientes en quienes se indicó un abordaje anterior complementario y aquellos es los que no, se observó que los pacientes con doble abordaje tenían una cifosis preoperatoria $7^{\circ}$ mayor en promedio y una altura corporal anterior $5 \mathrm{~mm}$ menor que aquellos sometidos solo a una estabilización percutánea. Esto concuerda con los criterios de indicación de un abordaje mínimamente invasivo por vía anterior asociado a la estabilización percutánea. En el último seguimiento, esta diferencia se redujo a $2^{\circ}$ de cifosis residual promedio (mayor cifosis residual en el grupo de percutáneo solo, $11^{\circ} \mathrm{vs} .9^{\circ}$ ) y a solo $1 \mathrm{~mm}$ de altura corporal vertebral promedio entre ambos grupos. 
Se ha estudiado y comparado la colocación precisa de los tornillos pediculares entre los procedimientos abiertos y percutáneos. Estos estudios muestran que la orientación percutánea del tornillo pedicular con guía fluoroscópica, cuando se utiliza una técnica adecuada, conduce a menos violaciones de la pared pedicular que cuando se realiza de manera abierta; el procedimiento percutáneo carece de sensación táctil, lo que aumenta aún más el desafío técnico y la dificultad asociados con la curva de aprendizaje empinada. ${ }^{36}$

En nuestra serie, la colocación fue excelente en el 91,7\%, 88 de 96 tornillos pediculares fueron colocados en posición 1A y 1B según Zdichavsky y cols..$^{21,22}$ (Figura 4) evaluados mediante TC posoperatoria. El 6,25\% ( 6 tornillos: dos 2A, cuatro 2B) tuvo una posición buena y solo dos (2\%) fueron colocados en posición 3B. No fue necesario reposicionar ningún tornillo.

Una vez que se produce la consolidación ósea, teóricamente, la instrumentación puede eliminarse, dado que los discos están intactos en este tipo de fracturas. Aunque no existe consenso, parece lógico eliminar la instrumentación de 8 a 12 meses después de que la fractura ha sido inmovilizada. Wild y cols. eliminaron la instrumentación en todos sus pacientes y hallaron una pérdida de la corrección durante el año posterior a la extracción, aunque esta pérdida se estabilizó con el tiempo. ${ }^{26}$ En los estudios, se hace poca mención a la extracción de tornillos pediculares, ya sean abiertos o percutáneos, después de la consolidación de la fractura. Esta pregunta aún no ha encontrado respuesta en estudios observacionales a largo plazo. En esta serie, fue necesario extraer la instrumentación en dos pacientes por molestias locales y por irritación radicular, a los 5 y a los 16 meses, respectivamente; antes de la cirugía se corroboró la consolidación de la fractura. Nuestro protocolo no incluye la extracción programada del implante.

El papel de la fijación espinal percutánea y mínimamente invasiva no reemplaza a las técnicas abiertas, sino que se agrega a las opciones terapéuticas. Los defensores de estas técnicas citan, entre otras, la reducción del tiempo operatorio, la menor pérdida de sangre y la disminución de la alteración de los tejidos blandos ya traumatizados. Por el contrario, quienes se oponen a la técnica mínimamente invasiva citan la larga curva de aprendizaje del cirujano y la posibilidad de una restauración inadecuada de la altura del cuerpo de las vértebras y de la cifosis local. Knox y cols. ${ }^{37}$ Patel y cols. ${ }^{38}$ y Park y cols.$^{39}$ comentaron sobre los desafíos a los que se enfrentaron en los primeros casos realizados en sus centros asistenciales. Reportan una mayor incidencia de complicaciones por la colocación incorrecta de tornillos, la violación de articulaciones facetarias y la necesidad subsiguiente de procedimientos operatorios adicionales. En nuestra experiencia, las complicaciones infecciosas se produjeron en el segundo y tercer paciente tratado y los dos únicos tornillos colocados en mala posición (3B) fueron en el primer caso.

Si bien es un estudio retrospectivo con una cantidad pequeña de pacientes, describe todos los procedimientos percutáneos y mínimamente invasivos que se realizaron en nuestro Servicio para las fracturas toracolumbares de causa traumática, representando la curva de aprendizaje en nuestro equipo. A pesar de que el número de la muestra no tiene el poder suficiente para crear datos con relevancia estadística, hemos logrado parear los resultados de las mediciones pre y posoperatorias de la cifosis segmentaria y la altura vertebral, y obtener información que favorece el empleo de estas técnicas. Destacamos que la mitad de nuestros pacientes fueron tratados quirúrgicamente a pesar de que el TLICSS resultara $<4$. Este es un punto controversial, porque la evidencia disponible ${ }^{27}$ no es concluyente sobre el patrón de referencia terapéutico para las fracturas tipo estallido sin compromiso neurológico.

Creemos necesario continuar la evaluación de esta serie a largo plazo, prestando especial atención a la necesidad del retiro del implante para así poder definir, sobre la base de la evidencia, nuestro protocolo de trabajo con estas técnicas mínimamente invasivas.

\section{CONSIDERACIONES FINALES}

El tratamiento de las fracturas toracolumbares de causa traumática mediante técnicas mínimamente invasivas fue un procedimiento seguro, con buenos resultados clínicos y radiológicos al final del seguimiento. Los tiempos de rehabilitación y estancia hospitalaria fueron comparables a los de otros centros asistenciales, aunque nuestra tasa de complicaciones fue más alta.

Somos optimistas en el uso de estas técnicas para tratar fracturas toracolumbares seleccionadas y lograr reproducir las ventajas reportadas por otros autores.

Conflicto de intereses: Los autores no declaran conflictos de intereses. 
ORCID de G. Camino Willhuber: https://orcid.org/0000-0002-5684-7679 ORCID de M. M. Estefan: https://orcid.org/0000-0002-7327-8703

ORCID de G. R. Kido: https://orcid.org/0000-0001-5521-7918

ORCID de J. Bassani: https://orcid.org/0000-0002-3340-8606
ORCID de M. G. Petracchi: https://orcid.org/0000-0001-7350-1816

ORCID de C. A. Sola: https://orcid org/0000-0001-9606-2107

ORCID de M. Gruenberg: https://orcid.org/0000-0001-7681-7058

\section{BIBLIOGRAFÍA}

1. Hu R, Mustard CA, Burns C. Epidemiology of incident spinal fracture in a complete population. Spine 1996;21(4):492-9. https://doi.org/ 10.1097/00007632-199602150-00016

2. Diaz Jr JJ, Cullinane DC, Altman DT, Bokhari F, Cheng JS, Como J, et al. Practice management guidelines for the screening of thoracolumbar spine fracture. J Trauma 2007;63(3):709-18. https://doi.org/10.1097/TA.0b013e318142d2db

3. Esses SI, Botsford DJ, Kostuik JP. Evaluation of surgical treatment for burst fractures. Spine (Phila Pa 1976) 1990;15(7):667-73. https://doi.org/10.1097/00007632-199007000-00010

4. Park Y, Ha JW. Comparison of one-level posterior lumbar interbody fusion performed with a minimally invasive approach or a traditional open approach. Spine 2007;32(5):537-43. https://doi.org/10.1097/01.brs.0000256473.49791.f4

5. Kim DY, Lee SH, Chung SK, Lee HY. Comparison of multifidus muscle atrophy and trunk extension muscle strength: percutaneous versus open pedicle screw fixation. Spine (Phila Pa 1976) 2005;30(1):123-9. PMID: 15626992

6. Rampersaud YR, Annand N, Dekutoski MB. Use of minimally invasive surgical techniques in the management of thoracolumbar trauma: current concepts. Spine 2006;31:S96-S102. https://doi.org/10.1097/01.brs.0000218250.51148.5b

7. Weber BR, Grod D, Dvorak J, Müntener M. Posterior surgical approach to the lumbar spine and its effect on the multifidus muscle. Spine (Phila Pa 1976) 1997;22:1765-72. https://doi.org/10.1097/00007632-199708010-00017

8. Ntilikina Y, Bahlau D, Garnon J, Schuller S, Walter A, Schaeffer M, et al. Open versus percutaneous instrumentation in thoracolumbar fractures: magnetic resonance imaging comparison of paravertebral muscles after implant removal. J Neurosurg Spine 2017;27(2):235-41. https://doi.org/10.3171/2017.1.SPINE16886

9. Phan K, Rao JP, Mobbs RJ. Percutaneous versus open pedicle screw fixation for treatment of thoracolumbar fractures: Systematic review and meta-analysis of comparative studies. Clin Neurol Neurosurg 2015;135:85-92. https://doi.org/10.1016/j.clineuro.2015.05.016

10. Foley KT, Gupta SK, Justis JR, Sherman MC. Percutaneous pedicle screw fixation of the lumbar spine. Neurosurg Focus 2001;10(4):E10. https://doi.org/10.3171/foc.2001.10.4.11

11. Mobbs RJ, Sivabalan P, Li J. Minimally invasive surgery compared to open spinal fusion for the treatment of degenerative lumbar spine pathologies. J Clin Neurosci 2012;19(6):829-35.

https://doi.org/10.1016/j.jocn.2011.10.004

12. Song HP, Lu JW, Liu H, Zhang C. [Case-control studies between two methods of minimally invasive surgery and traditional open operation for thoracolumbar fractures]. Zhongguo Gu Shang 2012;25(4):313-6. [En chino] PMID: 22812097

13. Court C, Vincent C. Percutaneous fixation of thoracolumbar fractures: current concepts. Orthop Traumatol Surg Res 2012;98(8):900-9. https://doi.org/10.1016/j.otsr.2012.09.014

14. Schmidt OI, Strasser S, Kaufmann V, Strasser E, Gahr RH. Role of early minimal-invasive spine fixation in acute thoracic and lumbar spine trauma. Indian J Orthop 2007;41(4):374-80. https://doi.org/10.4103/0019-5413.37003

15. Gruenberg MG, Solá CA, Petracchi MG, Ortolán EG. Fracturas por estallido en la columna dorsolumbar instrumentadas con tornillos pediculares. Rev Asoc Arg Ortop Traumatol 2003;68(4):273-83. https://www.aaot.org.ar/revista/2003/n4_vol68/art4.pdf

16. Nicolino TI, Petracchi M, Sola C, Valacco M, Gruenberg M. Evaluación clínica y radiológica de la instrumentación y artrodesis corta por vía posterior en fracturas toracolumbares. Rev Asoc Arg Ortop Traumatol 2015;80(1):20-29. https://doi.org/10.15417/267

17. Payares K, Lugo LH, Morales V, Londoño A. Validation in Colombia of the Oswestry disability questionnaire in patients with low back pain. Spine (Phila Pa 1976) 2011;36(26):E1730-5.

https://doi.org/10.1097/BRS.0b013e318219d184 
18. Frankel HL, Hancock PO, Hyslop G, Melzak J, Michaelis LS, Ungar GH, et al. The value of postural reduction in the initial management of closed injuries of the spine with paraplegia and tetraplegia. Part I. Paraplegia 1969;7:17992. https://doi.org/10.1038/sc.1969.30

19. Magerl F, Aebi M, Gertzbein SD, Harms J, Nazarian S. A comprehensive classification of thoracic and lumbar injuries. Eur Spine J 1994;3(4):184-201. https://doi.org/10.1007/BF02221591

20. Vaccaro AR, Lim MR, Hurlbert RJ, Lehman RA Jr, Harrop J, Fisher DC, et al. Surgical decision making for unstable thoracolumbar spine injuries: results of a consensus panel review by the Spine Trauma Study Group. $J$ Spinal Disord Tech 2006;19:1-10. https://doi.org/10.1097/01.bsd.0000180080.59559.45

21. Zdichavsky M, Blauth M, Knop C, Graessner M, Herrmann H, Krettek C, et al. Accuracy of pedicle screw placement in thoracic spine fractures. Part I: inter- and intra-observer reliability of the scoring system. Eur J Trauma 2004;30:234-240. https://doi.org/10.1007/s00068-004-1422-9

22. Zdichavsky M, Blauth M, Knop C, Lotz J, Krettek C, Bastian L. Accuracy of pedicle screw placement in thoracic spine fractures. Part II: a retrospective analysis of 278 pedicle screws using computed tomographic scans. Eur J Trauma 2004;30:241-7. https://doi.org/10.1007/s00068-004-1423-8

23. Pereira Duarte M, Petracchi MG, Mereles ME, Gruenberg M, Solá CA, Girardi FP. Artrodesis intersomática lateral de columna lumbar. Técnica quirúrgica y conceptos actuales. Rev Asoc Argent Ortop Traumatol 2018;83(4):303316. https://doi.org/10.15417/issn.1852-7434.2018.83.4.754

24. Alanay A, Pekmezci M, Karaemigogullari O, Acaroglu E, Yazici M, Cil A, et al. Radiographic measurement of the sagittal plane deformity in patients with osteoporotic spinal fractures evaluation of intrinsic error. Eur Spine J 2007;16(12):2126-32. https://doi.org/10.1007/s00586-007-0474-z

25. Assaker R. Minimal access spinal technologies: state-of-the-art, indications, and techniques. Joint Bone Spine 2004;71(6):459-69. https://doi.org/10.1016/j.jbspin.2004.08.006

26. Wild MH, Glees M, Plieschnegger C, Wenda K. Five-year follow-up examination after purely minimally invasive posterior stabilization of thoracolumbar fractures: a comparison of minimally invasive percutaneously and conventionally open treated patients. Arch Orthop Trauma Surg 2007;127(5):335-43. https://doi.org/10.1007/s00402-006-0264-9

27. Pehlivanoglu T, Akgul T, Bayram S, Meric E, Ozdemir M, Korkmaz M, et al. Conservative versus operative treatment of stable thoracolumbar burst fractures in neurologically intact patients: is there any difference regarding the clinical and radiographic outcomes? Spine (Phila Pa 1976) 2020;45(7):452-8. https://doi.org/10.1097/BRS.0000000000003295

28. Koosha M, Nayeb Aghaei H, Khayat Kashani HR, Paybast S. Functional outcome of surgical versus conservative therapy in patients with traumatic thoracolumbar fractures and thoracolumbar injury classification and severity score of 4; A non-randomized clinical rrial. Bull Emerg Trauma 2020;8(2):89-97. https://doi.org/10.30476/BEAT.2020.46448

29. McCormack T, Karaikovic E, Gaines RW. The load sharing classification of spine fractures. Spine (Phila Pa 1976) 1994;19(15):1741-4. https://doi.org/10.1097/00007632-199408000-00014

30. Ni WF, Huang YX, Chi YL, Xu HZ, Lin Y, Wang XY, et al. Percutaneous pedicle screw fixation for neurologic intact thoracolumbar burst fractures. J Spinal Disord Tech 2010;23:530-7. https://doi.org/10.1097/BSD.0b013e3181c72d4c

31. Merom L, Raz N, Hamud C, Weisz I, Hanani A. Minimally invasive burst fracture fixation in the thoracolumbar region. Orthopedics 2009;32(4). https://doi.org/10.3928/01477447-20090401-03

32. Palmisani M, Gasbarrini A, Brodano GB, De Iure F, Cappuccio M, Boriani L, et al. Minimally invasive percutaneous fixation in the treatment of thoracic and lumbar spine fractures. Eur Spine J 2009;18:71-4. https://doi.org/10.1007/s00586-009-0989-6

33. Rechtine GR, Bono PL, Cahill D, Bolesta MJ, Chrin AM. Postoperative wound infection after instrumentation of thoracic and lumbar fractures. J Orthop Trauma 2001;15(8):566-9. https://doi.org/10.1097/00005131-200111000-00006

34. Korovessis P, Hadjipavlou A, Repantis T. Minimal invasive short posterior instrumentation plus balloon kyphoplasty with calcium phosphate for burst and severe compression lumbar fractures. Spine (Phila Pa 1976) 2008;33(6):65867. https://doi.org/10.1097/BRS.0b013e318166e0bb

35. Pelegri C, Benchikh El Fegoun A, Winter M, Brassart N, Bronsard N, Hovorka I, et al. Percutaneous fixation of lumbar and thoracolumbar fractures without neurological involvement: surgical technique and preliminary results. Rev Chir Orthop Reparatrice Appar Mot 2008;94(5):456-63. https://doi.org/10.1016/j.rco.2008.03.035 
36. Rampersaud YR, Foley KT, Shen AC, Williams S, Solomito M. Radiation exposure to the spine surgeon during fluoroscopically assisted pedicle screw insertion. Spine (Phila Pa 1976) 2000;25(20):2637-45. https://doi.org/10.1097/00007632-200010150-00016

37. Knox JB, Dai JM III, Orchowski JR. Superior segment facet joint violation and cortical violation after minimally invasive pedicle screw placement. Spine J 2011;11(3):213-7. https://doi.org/10.1016/j.spinee.2011.01.024

38. Patel RD, Graziano GP, Vanderhave KL, Patel AA, Gerling MC. Facet violation with the placement of percutaneous pedicle screws. Spine (Phila Pa 1976) 2011;36(26):E1749-E1752. https://doi.org/10.1097/BRS.0b013e318221a800

39. Park Y, Ha JW, Lee YT, Sung NY. Cranial facet joint violations by percutaneously placed pedicle screws adjacent to a minimally invasive lumbar spinal fusion. Spine J 2011;11(4):295-302. https://doi.org/10.1016/j.spinee.2011.02.007 